\title{
Neurocognitive assessment of emotional context sensitivity
}

\author{
Sarah Myruski ${ }^{1,2} \cdot$ George A. Bonanno $^{3} \cdot$ Olga Gulyayeva $^{4,2} \cdot$ Laura J. Egan $^{5}$ • \\ Tracy A. Dennis-Tiwary ${ }^{2,1}$
}

Published online: 21 August 2017

(C) Psychonomic Society, Inc. 2017

\begin{abstract}
Sensitivity to emotional context is an emerging construct for characterizing adaptive or maladaptive emotion regulation, but few measurement approaches exist. The current study combined behavioral and neurocognitive measures to assess context sensitivity in relation to self-report measures of adaptive emotional flexibility and well-being. Sixty-six adults completed an emotional go/no-go task using happy, fearful, and neutral faces as go and no-go cues, while EEG was recorded to generate event-related potentials (ERPs) reflecting attentional selection and discrimination (N170) and cognitive control (N2). Context sensitivity was measured as the degree of emotional facilitation or disruption in the go/no-go task and magnitude of ERP response to emotion cues. Participants self-reported on emotional flexibility, anxiety, and depression. Overall participants evidenced emotional context sensitivity, such that when happy faces were go stimuli, accuracy improved (greater behavioral facilitation), whereas when fearful faces were no-go stimuli, errors
\end{abstract}

Electronic supplementary material The online version of this article (doi:10.3758/s13415-017-0533-9) contains supplementary material, which is available to authorized users.

Tracy A. Dennis-Tiwary

tracy.dennis@hunter.cuny.edu

Sarah Myruski

sbabkirk@gradcenter.cuny.edu

1 The Graduate Center, The City University of New York, New York, NY, USA

2 Hunter College, The City University of New York, New York, NY, USA

3 Teachers College, Columbia University, New York, NY, USA

4 St. John's University, Queens, NY, USA

5 St. Francis College, Brooklyn, NY, USA increased (disrupted behavioral inhibition). These indices predicted emotional flexibility and well-being: Greater behavioral facilitation following happy cues was associated with lower depression and anxiety, whereas greater disruption in behavioral inhibition following fearful cues was associated with lower flexibility. ERP indices of context sensitivity revealed additional associations: Greater N2 to fear go cues was associated with less anxiety and depression, and greater N2 and N170 to happy and fear no-go cues, respectively, were associated with greater emotional flexibility and well-being. Results suggest that pleasant and unpleasant emotions selectively enhance and disrupt components of context sensitivity, and that behavioral and ERP indices of context sensitivity predict flexibility and well-being.

Keywords Event related potential (ERP) $\cdot$ Regulatory flexibility $\cdot$ Emotion $\cdot$ Context sensitivity

Emotion regulation (ER) strategies, which allow individuals to modulate the experience and expression of emotions, are often characterized as adaptive versus maladaptive (e.g., John $\&$ Gross, 2004). For example, suppression of unpleasant emotions has been discussed as a relatively maladaptive strategy, while reappraisal, the reinterpretation of a stimulus or emotional response in a positive way, is considered relatively adaptive. However, while reappraisal has been shown to result in decreased subjective and physiological responses to unpleasant stimuli (Gross \& Levenson, 1993; Urry, 2009), it is less effective in highly intense emotional situations (Sheppes, Catran, \& Meiran, 2009). Furthermore, a meta-analysis examining the efficacy of distinct ER strategies to modulate emotion across a range of methods and contexts showed small to modest effect sizes (Webb, Miles, \& Sheeran, 2012).

In a recent review of this literature, Bonanno and Burton (2013, p. 591) referred to the assumption that strategies are 
dichotomously adaptive or maladaptive as the "fallacy of uniform efficacy." The available literature, they concluded, indicated instead that the effectiveness of any particular regulatory strategy will vary across people and situations and consequently that effective regulation must by necessity be flexible (Bonanno, Papa, Lalande, Westphal, \& Coifman, 2004; Chang, 2001; Kashdan \& Rottenberg, 2010). For example, different situational contexts will tend to evoke different and sometimes competing regulatory goals (Tamir, 2009). Some contexts are more demanding than others, and some behaviors and strategies are more adaptive in specific contexts or situations and less adaptive in others (Aldao, 2013).

Extending this work, a growing body of research has documented the important role of contextual discrimination in adaptive self-regulation. Sensitivity to contextual cues allows for optimal choice of regulatory strategy (Bonanno \& Burton, 2013), while the failure to respond appropriately to contextual cues, or context insensitivity, has been implicated in psychopathology (Diminich \& Bonanno, 2014; Rottenberg, Gross, \& Gotlib, 2005; Rottenberg, Kasch, Gross, \& Gotlib, 2002).

One reason that the ability to read and respond to changing contextual cues is so crucial is that the output of this process informs all other downstream aspects of flexible self-regulation. For example, the ability to utilize different types of regulatory responses, or repertoire (Aldao, 2013; Bonanno \& Burton, 2013), has shown positive associations to both health and well-being and to the capacity to adapt to highly stressful and potentially traumatic life events (Bonanno et al., 2004; Bonanno, Westphal, \& Mancini, 2011; Gupta \& Bonanno, 2011; Park, Chang, \& You, 2015; Westphal, Seivert, \& Bonanno, 2010). A long-standing tenant of emotion regulation theory, however, holds that the enactment of different types of strategies can only be effective if the selected strategies are matched to changing contextual demands and opportunities (Aldao, 2013; Cole, Martin, \& Dennis, 2004; Davidson, Putnam, \& Larson, 2000).

Given the primacy of context sensitivity, it is surprising that few studies have examined its features or their relation to other components of flexible self-regulation. The current study sought to address this gap by examining complementary aspects of context sensitivity during an emotional go/no-go task. Specifically, we measured both event-related potentials (ERPs), that capture sensitivity to emotional context with high-functional and temporal specificity, and performancerelated behavioral metrics, such as response time and performance accuracy. In addition, we also examined how these indices related to key downstream components of regulatory flexibility, specifically, the capacity to modulate emotional expression and suppression or expressive flexibility, and the ability to utilize different types of coping strategies, or coping flexibility, that have proven highly relevant to well-being and adaptive functioning.
The emotional go/no-go task (Tottenham, Hare, \& Casey, 2011 ) is well-suited to generate measures of context sensitivity because it requires discrimination of relevant stimuli and cognitive control over behavior within shifting contextual demands. In a standard go/no-go task (Casey et al., 1997; Schulz et al., 2007) participants are required to indicate by button press whenever a designated target appears ("go" response), and to inhibit that response when a designated nontarget appears ("no-go" response). Because go trials occur with high frequency (e.g., 70\% of trials), response inhibition on the nogo trials requires cognitive control. A recent version of this task, using facial expressions of emotion as target stimuli, the emotional go/no-go task (Schulz et al., 2007; Tottenham et al., 2011), provides a means of assessing both basic contextual discrimination and control and emotion-related contextual discrimination and control within a single experimental design. Greater accuracy and faster reaction times to go trials represent successful behavioral facilitation toward the target, while lower false-alarm rates to no-go trials represent successful behavioral inhibition. Emotion modulates behavioral facilitation and inhibition (e.g., Ladouceur et al., 2006; Tottenham et al., 2011). Approach is facilitated when happy faces are the go stimulus, and inhibition is disrupted when emotional faces are the no-go stimulus (Tottenham et al., 2011), although it remains unclear how success or failure in varying emotional contexts are related to other components of regulatory flexibility.

Individual differences in the impact of emotion on approach or withdrawal behavior may predict ER flexibility and emotional well-being. For example, in comparison to typical controls, individuals diagnosed with anxiety disorders responded slower to go stimuli when the no-go cues were angry faces, indicating reduced behavioral facilitation in an emotionally unpleasant context (Ladouceur et al., 2006). Consistent with dual-process models (Carver \& White, 1994; Gray \& Braver, 2002), this suggests that avoidance-related emotions can selectively disrupt inhibitory control, whereas approach-related emotions can selectively promote behavioral facilitation. Importantly, the distinction between a normative disruption of inhibitory control by unpleasant emotions and an interference indicative of psychological dysfunction is not well understood. Likely, the behavioral performance differences between normative and disordered patterns of response are likely not categorical, and instead vary continuously. That is, slower behavioral facilitation and impaired inhibitory control to unpleasant stimuli may be normative when the magnitude of the effect is moderate (e.g., negative affective stimuli disrupting go/no-go latency) but when more extreme, may be linked to dysfunction. We attempt to clarify this difference in the current study by measuring contextual sensitivity as the influence of emotional information on cognitive control performance and 
testing whether metrics of this influence in turn is related to individual differences in self-reported well-being and adaptive regulatory flexibility.

Event-related potentials (ERPs) provide a more direct method for assessing sensitivity to emotional context. The N170 component is maximal at right lateralized occipitotemporal recording sites at 150 to $180 \mathrm{~ms}$ after stimulus onset (Bentin, Allison, Puce, Perez, \& McCarthy, 1996) and is thought to reflect relatively early attentional selection and discrimination, particularly for facial stimuli (Batty \& Taylor, 2003; Eimer, 2011). This component is thought to be generated in the superior temporal sulcus and fusiform gyrus, regions responsible for facial processing (Itier \& Taylor, 2004; Pizzagalli et al., 2002). The N170 is sensitive to emotion (e.g., Ashley, Vuilleumier, \& Swick, 2004; Batty \& Taylor, 2003; Pizzagalli et al., 2002), with greater amplitudes to emotional versus neutral stimuli, specifically fearful and angry faces (e.g., Batty \& Taylor, 2003; Krombholz, Schaefer, \& Boucsein, 2007). In emotional go/no-go tasks, N170 amplitudes are greater to emotional versus neutral faces, particularly for no-go trials, when attentional selection and discrimination is essential (Wang, Nie, \& Lu, 2014). However, greater N170 amplitudes to emotional no-go faces were linked to reduced response inhibition, suggesting that the resource priority given to the early processing of emotional content may disrupt subsequent behavioral inhibition (Wang et al., 2014). N170s also reflect dysfunctional processing of emotion in individuals with psychiatric diagnoses, including depression (see Feuerriegel, Churches, Hofmann, \& Keage, 2015, for a review).

The N2 component is thought to reflect later, higher level cognitive control (Van Veen \& Carter, 2002) and is a negative shift, which is maximal at frontal recording sites approximately 200 to $300 \mathrm{~ms}$ after stimulus onset. The N2 reflects activation of frontal lobe (e.g., Falkenstein, Hoormann, \& Hohnsbein, 1999; Kiefer, Marzinzik, Weisbrod, Scherg, \& Spitzer, 1998; Kopp, Mattler, Goertz, \& Rist, 1996) and anterior cingulate cortex (Bokura, Yamaguchi, \& Kobayashi, 2001), regions involved in executive control of motor responses and emotion regulation (see Bush, Luu, \& Posner, 2000, for a review). Greater N2 amplitudes coincide with successful response inhibition for no-go trials (Falkenstein et al., 1999), potentially indicating increased resource allocation to effortful inhibition of motor responses (Geczy, Czigler, \& Balazs, 1998). Furthermore, N2 amplitudes are attenuated following no-go trials among individuals with impulse control problems such as ADHD and addiction (Dong, Lu, Zhou, \& Zhao, 2010; Pliszka, Liotti, \& Woldorff, 2000).

We examined these ERPs in the current study as part of a complementary, multimethod approach to measure emotional context sensitivity (facilitation and inhibition) and examine it in relation to measures of adaptive emotion regulation and regulatory flexibility. Behaviorally, happy stimuli are predicted to bolster behavioral facilitation whereas fear stimuli are predicted to disrupt behavioral inhibition. On a physiological level, N170 amplitudes are predicted to be greater to emotional versus neutral stimuli, indicating emotional enhancement of attentional selection and discrimination, whereas N2 amplitudes are predicted to be reduced, reflecting taxing of cognitive control to inhibit responses to emotional no-go stimuli.

In addition, we examined links between biobehavioral measures of emotional context sensitivity in relation to selfreported adaptive emotion regulation (emotional well-being and flexibility). We predicted that individuals reporting more positive emotional well-being and greater regulatory flexibility would also show behavioral and physiological indices of greater ability to harness the bolstering effects of pleasant emotions on behavioral facilitation as well as greater resistance to the interfering effects of unpleasant emotion on behavioral inhibition.

\section{Method}

\section{Participants}

Sixty-six adults ( 17 males, 49 females), ages 18 to 47 years ( $M$ $=20.97$ years, $S D=4.76$ ), participated in the current study. ${ }^{1}$ Participants were recruited through the psychology participant pool at Hunter College, The City University of New York. Self-reported race/ethnicity included two African American (3.0\%), 12 Hispanic (18.2\%), 26 Caucasian (39.4\%), 17 Asian (25.8\%), and nine (13.6\%) other.

\section{Materials and procedure}

Following informed consent, participants completed various questionnaires. After the questionnaire period, EEG electrodes were applied, and participants were seated in an EEG recording booth $65 \mathrm{~cm}$ from a 17 -in. monitor to complete an emotional go/no-go task. Participants spent a total of approximately 2 hours in the laboratory.

EEG recording and data reduction A Biosemi system (BioSemi; Amsterdam, Netherlands), was used to record EEG activity continuously during the emotional go/no-go task using $64 \mathrm{Ag} / \mathrm{AgCl}$ scalp electrodes. Electrodes were fixed into an elasticized nylon cap and arranged according to the International 10-20 system. Eye movements were monitored by electrooculogram (EOG) signals from electrodes placed $1 \mathrm{~cm}$ above and below the left eye (to measure vertical eye

\footnotetext{
${ }^{1}$ A total of 77 participants were recruited; three were excluded due to difficulties during EEG data collection, and an additional eight were excluded due to low trial counts ( $<5$ trials for no-go).
} 
movements) and $1 \mathrm{~cm}$ on the outer edge of each eye (to measure horizontal eye movements). Preamplification of the EEG signal occurred at each electrode which improves the signalto-noise ratio. EEG was recorded at a sampling rate of $512 \mathrm{~Hz}$. During EEG acquisition, the voltage from each of the 64 electrodes from which data were collected was referenced online with respect to the common mode sense active electrode and driven right leg electrode, which produces a monopolar (nondifferential) channel. Brain Vision Analyzer (Version 2.2, $\mathrm{GmbH}$; Munich, Germany) was used to prepare the data. Offline, all data were rereferenced to the average of the scalp and filtered with a high-pass frequency of $0.1 \mathrm{~Hz}$ and a lowpass frequency of $30 \mathrm{~Hz}$. Face-locked data were segmented into epochs from $200 \mathrm{~ms}$ before stimulus presentation to $500 \mathrm{~ms}$ after stimulus onset, with a 200-ms baseline correction separately for the go and no-go faces. The N170 was generated as the mean amplitude from $140 \mathrm{~ms}$ to $190 \mathrm{~ms}$ over P9 and $\mathrm{P} 10$, and $\mathrm{N} 2$ was generated as the mean amplitude from $220 \mathrm{~ms}$ to $320 \mathrm{~ms}$ over Fz (see Fig. 1).

Emotional go/no-go task Emotional faces (fearful, happy, neutral) from the NimStim set (Tottenham et al., 2009) were presented as either the go or no-go stimulus. Stimuli were presented on-screen for $500 \mathrm{~ms}$ with a 1,500 -ms intertrial interval (ITI). Each condition consisted of 50 trials: 35 go trials $(70 \%)$ and 15 no-go trials (30\%). There were a total of six conditions, each consisting of emotion-neutral contexts (fear-neutral, happy-neutral) or mixed emotion contexts (fear-happy). Within each condition, one face type was assigned to be the go stimulus and another to be the no-go stimulus as follows: (fear go/neutral no-go, fear no-go/neutral go, happy go/neutral no-go, happy no-go/neutral go, fear go/ happy no-go, fear no-go/happy go). Condition order and face pair-presentation order within conditions were

\section{N170 to Go stimuli}
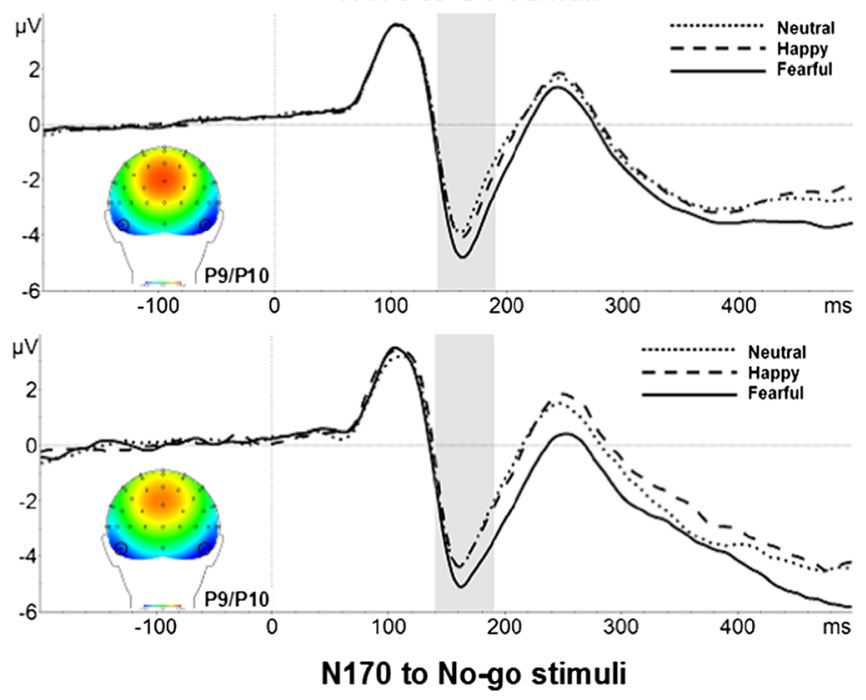

counterbalanced across participants. Conditions were organized into three blocks for analyses: fear-neutral (includes fear go/neutral no-go, fear no-go/neutral go); happy-neutral (includes happy go/neutral no-go, happy no-go/neutral go); and fear-happy (including fear go/happy no-go, fear no-go/happy go). Thus, the term block describes which emotions are present in the context (e.g., fear-neutral block includes fear go/ neutral no-go, fear no-go/neutral go).

Participants were instructed to respond to the go stimulus (one face type; e.g., happy faces) in each block by pressing the spacebar as quickly as possible and to refrain from responding to the no-go stimulus (any face type other than the go stimulus; e.g., fear faces; see Fig. 2). Hit rates for correct responses to go trials (behavioral facilitation) as well as false-alarm rates for no-go trials (behavioral inhibition) were recorded. We calculated $d$ prime scores $(z$-transformed hit rate minus $z$-transformed false-alarm rate) to quantify accuracy while taking response bias into account.

To examine the variability attributable to emotional processing compared to its baseline emotion (e.g., fearful vs. neutral faces) within a block, residual scores were computed for each behavioral metric (e.g., hit rate) separately. Residuals are thought to be a more reliable method for measuring responses relative to a baseline emotion compared to subtraction scores since (a) subtraction scores do not account for intercorrelations between the baseline emotion and relative responses (Weinberg, Venables, Proudfit, \& Patrick, 2015), and (b) subtraction scores do not reflect differences in variability of measurement across individuals. Greater residual scores represent greater difference from baseline emotions because they reflect a larger response than what would be predicted by the respective baseline emotion model.

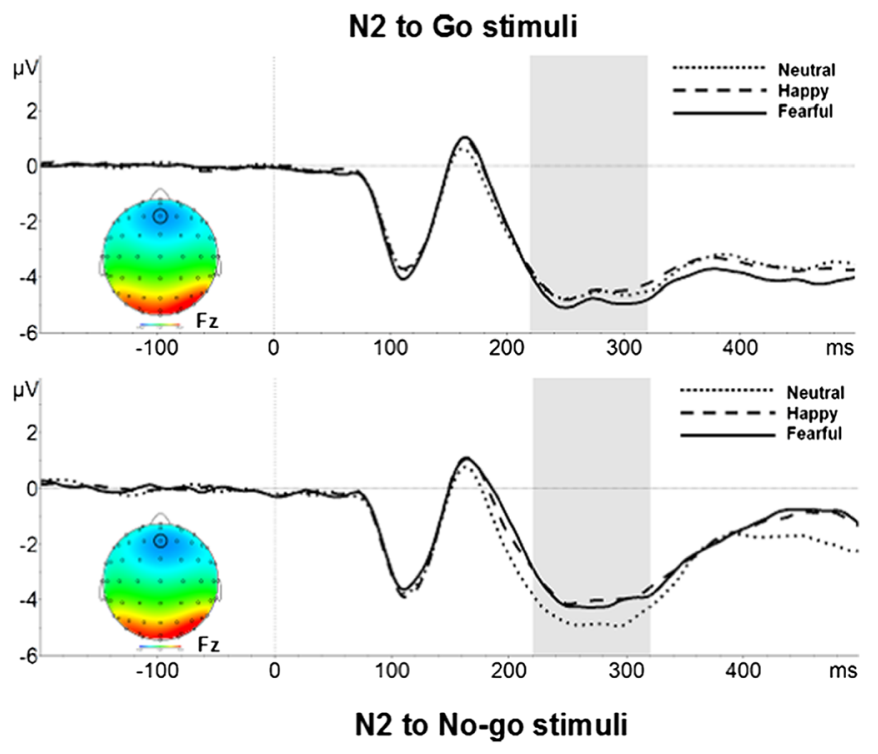

Fig. 1 Waveforms represent the N170 and N2 components for go and no-go stimuli, separately for neutral, happy, and fearful faces. (Color figure online) 


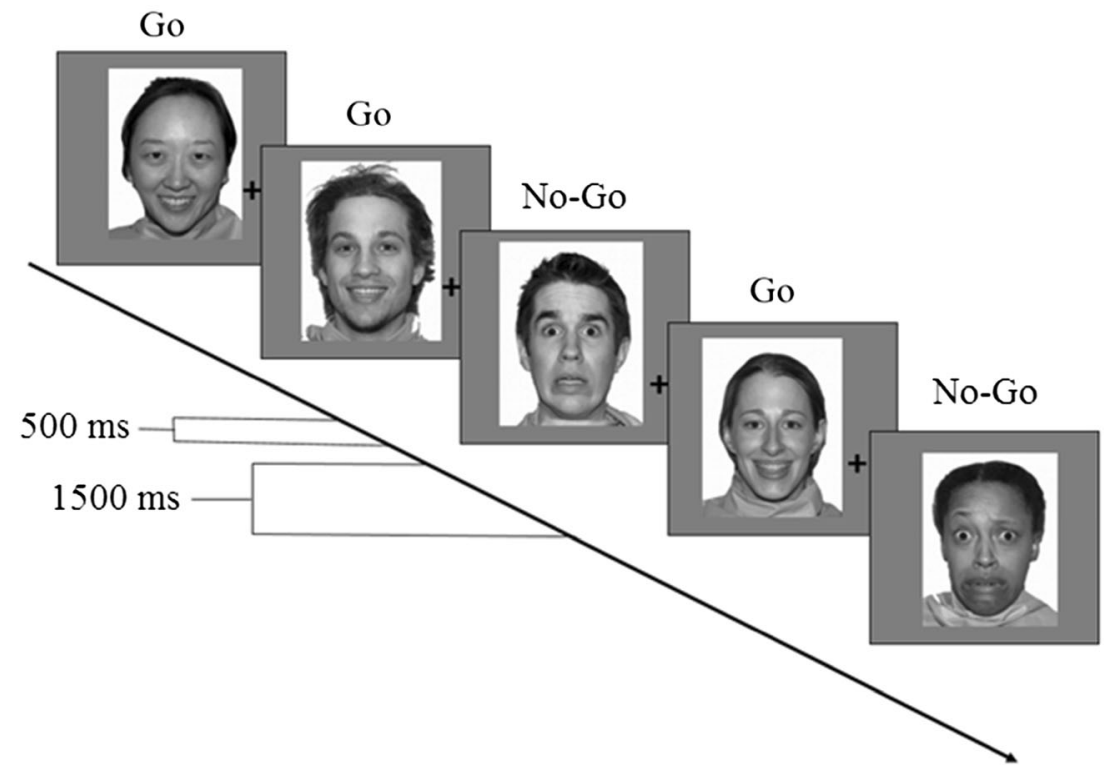

Fig. 2 Illustration of go/no-go task. In this example, happy faces are the go stimulus and fearful faces are the no-go stimulus. Adapted from Tottenham, Hare, and Casey (2011)

Residual scores were computed for hit and false-alarm rates as follows: For the fear-neutral block, responses to fear cues were predicted from responses to neutral cues; for the happy-neutral block, responses to happy cues were predicted from responses to neutral cues; and for the fearhappy block, responses to fear cues were predicted from responses to happy cues.

Residual scores were also computed for the N170 and $\mathrm{N} 2$ for each stimulus type separately (go and no-go) as follows: for the fear-neutral block, ERPs to fear cues were predicted from ERPs to neutral cues; for the happy-neutral block, ERPs to happy cues were predicted from ERPs to neutral cues; and for the fear-happy block, ERPs to fear cues were predicted from ERPs to happy cues.

\section{Self-report of regulatory flexibility and well-being}

Emotional well-being Participants completed two self-report measures of emotional well-being. the Beck Depression Inventory (BDI; Beck, Steer, \& Brown, 1996) is a 21-item questionnaire that has participants assesses depressive symptoms over the previous 2-week period $(\alpha=.93)$. The Beck Anxiety Inventory (BAI; Beck, Epstein, Brown, \& Steer, 1988 ) is a 21-item questionnaire that assesses general level of nervousness, anxiety, and shyness $(\alpha=0.92)$.

Emotion regulation and flexibility Participants completed self-report measures of emotion regulation and flexibility. The Flexible Regulation of Emotional Expression Scale (FREE; Burton \& Bonanno, 2015) measures the ability to modulate emotional experiences to pleasant and unpleasant information $(\alpha=.70-.81)$; and the Perceived Ability to
Cope with Trauma Scale (PACT; Bonanno, Pat-Horenczyk, $\&$ Noll, 2011) was used to measure coping flexibility in response to aversive or potentially traumatic events $(\alpha=$ $.79-.85)$.

\section{Results}

\section{Descriptive statistics}

Descriptive statistics for all self-report measures, behavioral performance measures, and ERP metrics are presented in Table 1. Since the current sample consisted of typical adults, we confirmed that depression (BDI) and anxiety (BAI) symptoms were normally distributed with means below the clinical ranges (see Table 1). One participant scored in the severe depression range $(>30)$ on the BDI, and eight participants scored in the severe anxiety range $(>25)^{2}$

\section{Metrics of emotional context sensitivity}

Analytic approach To examine behavioral and neurocognitive responses in varying emotional contexts, repeated-measures analyses of variance (ANOVA) were conducted. Emotion context effects on behavioral performance are reported below in the first section, and emotion context effects on neurocognitive responses are reported in the second

\footnotetext{
${ }^{2}$ All analyses were confirmed without participants scoring in the severe range for either depression or anxiety, and results did not differ from those from the full sample.
} 
Table 1 Descriptive statistics for self-report questionnaires and residuals indexing behavioral and neurocognitive performance

\begin{tabular}{|c|c|c|}
\hline & & Mean $(S D)$ \\
\hline \multicolumn{3}{|c|}{ Self-report questionnaires } \\
\hline \multicolumn{2}{|c|}{ Beck's Depression Inventory } & $11.60(8.45)$ \\
\hline \multicolumn{2}{|c|}{ Beck’s Anxiety Inventory } & $13.00(10.20)$ \\
\hline \multicolumn{2}{|c|}{ PACT Coping Flexibility } & $8.58(1.81)$ \\
\hline \multicolumn{2}{|c|}{ FREE Expressive Flexibility } & $60.39(13.14)$ \\
\hline \multicolumn{3}{|c|}{$D$-prime residuals } \\
\hline \multicolumn{2}{|c|}{ FN block } & $.00(1.42)$ \\
\hline \multicolumn{2}{|l|}{ HN block } & $.00(1.22)$ \\
\hline \multicolumn{2}{|l|}{ FH block } & $.00(1.45)$ \\
\hline \multicolumn{3}{|c|}{ Hit rate residuals } \\
\hline \multicolumn{2}{|c|}{ FN block } & $.00(.06)$ \\
\hline \multicolumn{2}{|l|}{ HN block } & $.00(.03)$ \\
\hline \multicolumn{2}{|l|}{ FH block } & $.00(.04)$ \\
\hline \multicolumn{3}{|c|}{ False-alarm rate residuals } \\
\hline \multicolumn{2}{|l|}{ FN block } & $.00(.11)$ \\
\hline \multicolumn{2}{|l|}{ HN block } & $.00(.11)$ \\
\hline \multicolumn{2}{|l|}{ FH block } & $.00(.10)$ \\
\hline \multicolumn{3}{|c|}{ N170 residuals } \\
\hline \multirow[t]{2}{*}{ FN block } & Fear no-go & $.00(2.84)$ \\
\hline & Fear go & $.00(2.04)$ \\
\hline \multirow[t]{2}{*}{ HN block } & Happy no-go & $.00(2.70)$ \\
\hline & Happy go & $.00(1.93)$ \\
\hline \multirow[t]{2}{*}{ FH block } & Fear no-go & $.00(2.89)$ \\
\hline & Fear go & $.00(1.98)$ \\
\hline \multicolumn{3}{|l|}{ N2 residuals } \\
\hline \multirow[t]{2}{*}{ FN block } & Fear no-go & $.00(2.72)$ \\
\hline & Fear go & $.00(2.12)$ \\
\hline \multirow[t]{2}{*}{ HN block } & Happy no-go & $.00(2.48)$ \\
\hline & Happy go & $.00(2.00)$ \\
\hline \multirow[t]{2}{*}{ FH flock } & Fear no-go & $.00(3.23)$ \\
\hline & Fear go & $.00(1.96)$ \\
\hline
\end{tabular}

Note. $\mathrm{FN}=$ fear-neutral block; $\mathrm{HN}=$ happy-neutral block; $\mathrm{FH}=$ fearhappy block

section. Within each of these two sections, two broad types of emotional comparisons were made.

First, to examine the influence of emotion versus neutral faces, the model compared the fear-neutral to happyneutral block, which were composed of all the emotionneutral conditions: fear go/neutral no-go, fear no-go/neutral go, happy go/neutral no-go, neutral go/happy no-go.

Second, to examine the influence of mixed emotion contexts (e.g. happy go/fear no-go), two models were tested: one model compared the fear-happy (fear go/ happy no-go and fear no-go/happy go conditions) to the fear-neutral block (fear go/neutral no-go, fear nogo/neutral go conditions) and the other model compared the fear-happy to the happy-neutral block (happy go/ neutral no-go, neutral go/happy no-go conditions). ${ }^{3}$

Behavioral performance First, we examined whether emotional faces would facilitate or disrupt behavioral performance relative to neutral faces, and whether this would differ depending on the broader affective context (e.g., whether fearful faces were paired with happy or neutral faces). First, $d$-prime scores were examined as the primary dependent measure of interest. Subsequently, hit rate and false-alarm rate were then investigated as secondary isolated indices of faciliatory and inhibitory processes, respectively. These secondary analyses were included to examine whether hit rate or false-alarm rate individually represented the driving force behind context sensitivity encompassed by $d$-prime. Repeated-measures analyses of variance were conducted separately for each behavioral measure ( $d$-prime, hit rate, false-alarm rate) as a dependent variable: The fear-neutral block was directly compared to the happyneutral block, 2 (block: fear-neutral, happy-neutral) $\times 2$ (valence: emotion, neutral); the fear-neutral block directly compared to the fear-happy block, 2 (block: fear-neutral, fear-happy) $\times 2$ (valence: fearful, nonfearful); and the happy-neutral block directly compared to the fear-happy block, 2 (block: happy-neutral, fear-happy) $\times 2$ (valence: happy, nonhappy) (see Supplement). Bonferroni correction (adjusted $p<$ .0083) was used to control for multiple comparisons in follow-up $t$ tests examining patterns of behavioral responding. 4

D-prime $D$-prime refers to the $z$-transformed response accuracy to go trials minus the $z$-transformed false-alarm rate for no-go trials. Greater scores indicated better context sensitivity such that participants were able to respond accurately to go stimuli while refraining from incorrectly responding to no-go stimuli.

Fear-neutral versus happy-neutral. D-prime was greater for emotional versus neutral faces, valence: $F(1,65)=$ 4.18, $p=.045, \eta_{\mathrm{p}}{ }^{2}=.06$; see Fig. 3, suggesting facilitation of context sensitivity by emotional cues. $D$-prime was greater for happy versus neutral go (happy-neutral block, $p=.001$ ) and fear go (across blocks, $p=.008$ ) faces, whereas no difference emerged in the fear-neutral

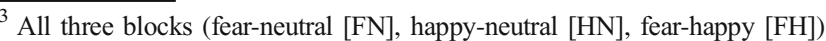
could not all be examined simultaneously in one repeated-measures ANOVA model for the following reason. Since the FH block is a mixed emotion block with no neutral faces, including this block in the same comparison with $\mathrm{HN}$ and FN would result in either the happy faces or the fearful faces being considered the neutral within-subjects variable in one block, but not in the corresponding $\mathrm{HN}$ or FN block. For this reason, blocks were examined in pairs (fear-neutral/happy-neutral, fear-neutral/fear-happy, and happy-neutral/fearhappy). See Supplement for further clarification.

${ }^{4}$ Bonferroni's correction for multiple comparisons was used in the analyses of covariance reported here, while the Behjamini-Hochberg correction was used for correlations reported below, since Benjamini-Hochberg is not recommended for within-subject tests (Benjamini \& Hochberg, 1995).
} 
Main Effect of Valence in the FN and FH Blocks

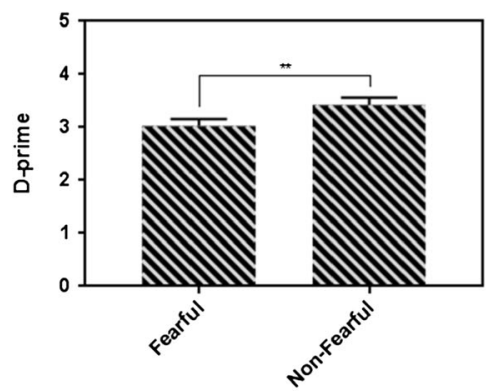

Main Effect of Valence in the $\mathrm{HN}$ and FH Blocks
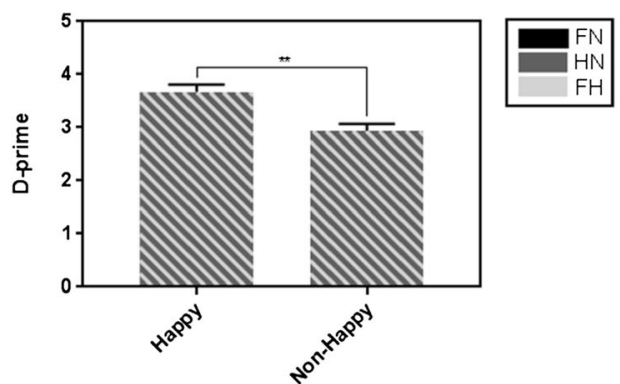

Fig. 3 Fearful faces disrupted context sensitivity such that $d$-prime was lower for fearful versus nonfear faces (top left). Context sensitivity was bolstered by emotional versus neutral faces (top right). More specifically, happy faces facilitated context sensitivity such that $d$-prime was greater

block $(p=.437)$; Block $\times$ Valence: $F(1,65)=8.02, p=$ $.006, \eta_{\mathrm{p}}^{2}=.11$.

Fear-neutral versus fear-happy. D-prime was lower for fearful versus non-fearful (happy and neutral) faces, valence: $F(1,65)=8.43, p=.005, \eta_{\mathrm{p}}{ }^{2}=.12$, suggesting disruption of context sensitivity by fearful face cues.

Happy-neutral versus fear-happy. D-prime was greater for happy versus non-happy (fearful and neutral) faces, valence: $F(1,65)=25.72, p<.001, \eta_{\mathrm{p}}{ }^{2}=.28$, suggesting facilitation of context sensitivity by pleasant cues.

Hit rate ${ }^{5}$ Hit rate refers to the number of times participants correctly responded to go trials, with greater rates indicating increased behavioral facilitation to the go stimulus.

Fear-neutral versus happy-neutral. Hit rates were greater when emotional versus neutral faces were the go stimulus, valence: $F(1,65)=7.82, p=.007, \eta_{\mathrm{p}}{ }^{2}=.11$. Hit rates were greater for happy versus neutral go faces (happy-neutral block: $p=$ .002 ), whereas no difference emerged in the fear-neutral block $(p=.464)$; Block $\times$ Valence: $F(1,65)=8.24, p=.006, \eta_{\mathrm{p}}{ }^{2}=.11$.

\footnotetext{
${ }^{5}$ Hit reaction times were also examined as an index of behavioral facilitation to the go stimulus, and the pattern of results exactly mirrored those found for hit rate. Thus, only hit rate results were included since they more appropriately coincide with the rate metric used for false alarms, and are a component of the $d$-prime score.
}

Main Effect of Valence in the FN and HN Blocks
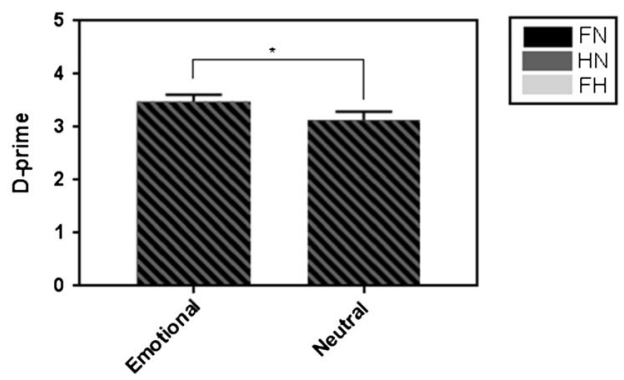

Block $X$ Valence Interaction across FN and HN Blocks

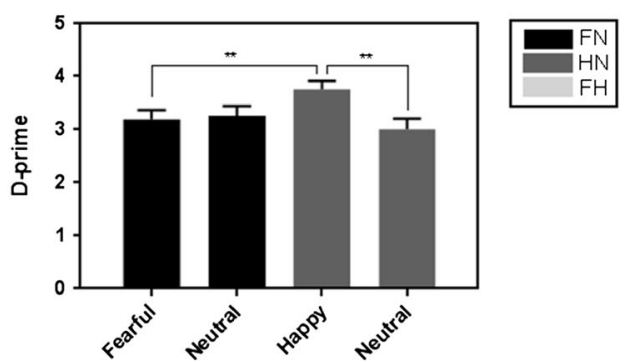

for happy faces compared to both fearful faces and neutral faces (bottom left, bottom right). Note. FN = fear-neutral block; $\mathrm{HN}=$ happy-neutral block; FH = fear-happy block

Fear-neutral versus fear-happy. Hit rates were greater for happy versus fearful faces (fear-happy block: $p<.001$ ), whereas no differences emerged in the fear-neutral block ( $p \mathrm{~s}>.008)$; Block $\times$ Valence: $F(1,65)=4.18, p=.045, \eta_{\mathrm{p}}^{2}=.06$.

Happy-neutral versus fear-happy. Hit rates were greater for happy go faces compared to neutral or fearful faces across and within blocks, valence: $F(1,65)=15.53, p<.001, \eta_{\mathrm{p}}{ }^{2}=.20$; Block $\times$ Valence: $F(1,65)=4.84, p=.031, \eta_{\mathrm{p}}^{2}=.07, p \mathrm{~s}<$ .008 .

False-alarm rate False-alarm rate refers to the number of times participants incorrectly responded to no-go trials, indicating a failure in behavioral inhibition.

Fear-neutral versus happy-neutral. False-alarm rates were greater to emotional versus neutral faces, valence: $F(1,65)=$ $4.06, p=.048, \eta_{\mathrm{p}}^{2}=.06$.

Fear-neutral versus fear-happy. There were no significant differences in false-alarm rates in the fear-neutral and fearhappy blocks $(p s>.10)$.

Happy-neutral versus fear-happy. There were no significant differences in false-alarm rates in the happy-neutral and fear-happy blocks ( $p$ s $>.10)$.

Behavioral performance summary Context sensitivity, measured as $d$-prime, was influenced by emotion such that $d$-prime 
Block X Valence Interaction across

FN and HN Blocks
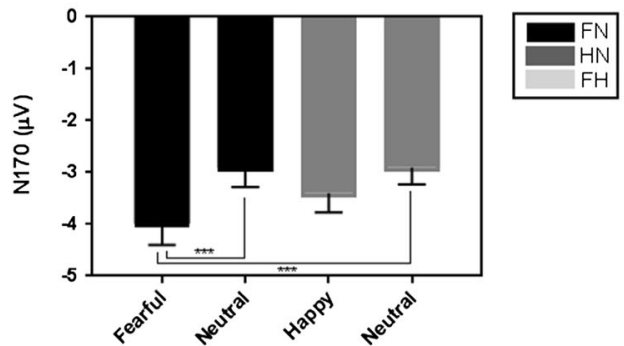

Block X Stimulus Interaction across FN and FH Blocks
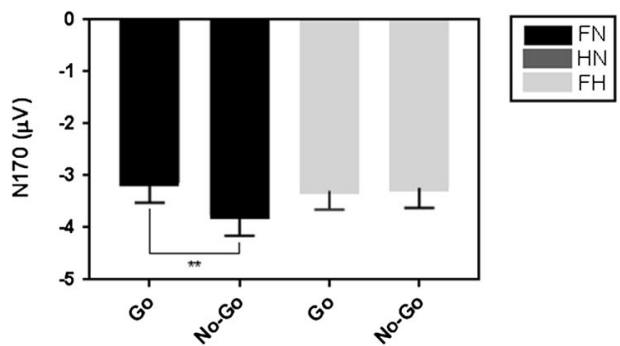

Fig. 4 The N170 was sensitive to emotional context: N170 amplitudes were larger to the fearful versus neutral (top left) and happy (top right) faces. Also, the N170 was greater to no-go versus go stimuli (bottom left,

was enhanced for happy faces and disrupted for fearful faces. Examination of hit rates and false-alarm rates revealed that happy faces bolstered behavioral facilitation (i.e., greater hit rates for happy vs. fearful and neutral faces), although emotional faces overall hindered behavioral inhibition (i.e., greater false-alarm rates for emotional vs. neutral faces).

ERP responses to face cues Next, we examined whether the N170 and N2 showed selective sensitivity to fearful, happy, or neutral faces, and whether this sensitivity differed by the broader emotional context. As described above for behavioral measures, three repeated-measures ANOVAs were conducted separately for each ERP measure (N170, N2) as dependent variable. The fearneutral block was directly compared to the happy-neutral block, 2 (block: fear-neutral, happy-neutral) $\times 2$ (valence: emotion, neutral) $\times 2$ (stimulus: go, no-go); the fear-neutral block directly compared to the fear-happy block, (block: fear-neutral, fear-happy) $\times 2$ (valence: fearful, non-fearful) $\times 2$ (stimulus: go, no-go); and the happyneutral block directly compared to the fear-happy block, 2 (block: happy-neutral, fear-happy) $\times 2$ (valence: happy, nonhappy) $\times 2$ (stimulus: go, no-go). ${ }^{6}$ Bonferroni correction (adjusted $p<.0083$ ) was used to control for multiple comparisons in follow-up $t$ tests examining patterns of neurocognitive responding.

\footnotetext{
${ }^{6}$ Since ERPs were generated for both go and no-go faces, stimulus could be included as a variable in these models. For models examining behavioral performance, measures were either indexing responses only to go trials ( $d$ prime, hit rate) or no-go trials (false-alarm rate), so stimulus could not be included in those models.
}

Block X Valence Interaction across HN and FH Blocks

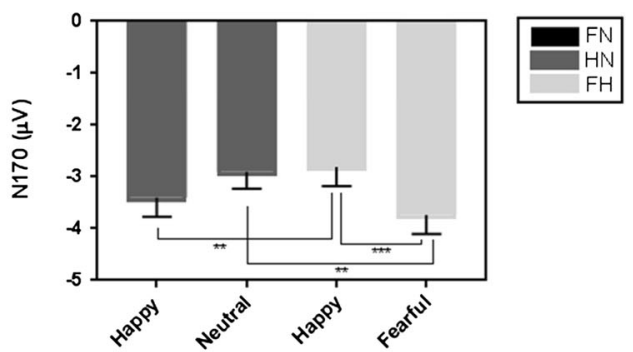

Block X Stimulus Interaction across HN and FH Blocks

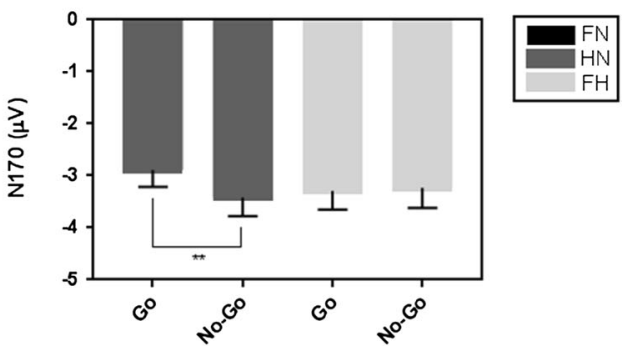

bottom right), but only in a context including neutral faces (fear-neutral or happy-neutral). Note. FN = fear-neutral block; $\mathrm{HN}=$ happy-neutral block; $\mathrm{FH}=$ fear-happy block

N170 Fear-neutral versus happy-neutral. There was a significant main effect of stimulus: N170 amplitudes were larger to no-go versus go faces, $F(1,65)=19.05, p<.001, \eta_{\mathrm{p}}{ }^{2}=.23$. In addition, the main effect of valence, showing that $\mathrm{N} 170$ amplitudes were larger to emotional versus neutral faces, $F(1,65)=33.74, p<.001, \eta_{\mathrm{p}}{ }^{2}$ $=.34$; was subsumed under a marginally significant interaction with block, Block $\times$ Valence: $F(1,65)=3.40, p=.070, \eta_{\mathrm{p}}{ }^{2}=.05$, such that N170 amplitudes were larger to both the fearful and happy faces relative to neutral, with fearful faces showing the largest magnitude $\mathrm{N} 170$ (all $p s<.01$; see Fig. 4).

Fear-neutral versus fear-happy. There was a main effect of valence such that N170 amplitudes were larger to fearful versus nonfearful faces, $F(1,65)=42.79, p<.001, \eta_{\mathrm{p}}{ }^{2}=.40$. Also, there was a main effect of stimulus such that that N170 amplitudes were larger to no-go versus go stimuli, $F(1,65)=5.16, p$ $=.026, \eta_{\mathrm{p}}{ }^{2}=.07$, but this difference was significant in the fearneutral block only, Block $\times$ Stimulus: $F(1,65)=6.68, p=.012$, $\eta_{\mathrm{p}}^{2}=.09 ; p=001$.

Happy-neutral versus fear-happy. There was a significant Block $\times$ Valence interaction such that N170 amplitudes were greater for fearful versus happy or neutral faces $(p s<.002)$, and greater for happy in a neutral context compared to a fearful context $(p=008), F(1,65)=34.27, p<.001, \eta_{\mathrm{p}}{ }^{2}=.35$. Also, a significant Block $\times$ Stimulus interaction showed that N170 amplitudes were greater for no-go versus go faces, but only in the happy-neutral block $(p=006), F(1,65)=5.22, p=.026, \eta_{\mathrm{p}}{ }^{2}$ $=.07$.

N170 Summary. N170 amplitudes were sensitive to stimulus, valence, and block, indicating greater attentional selection 

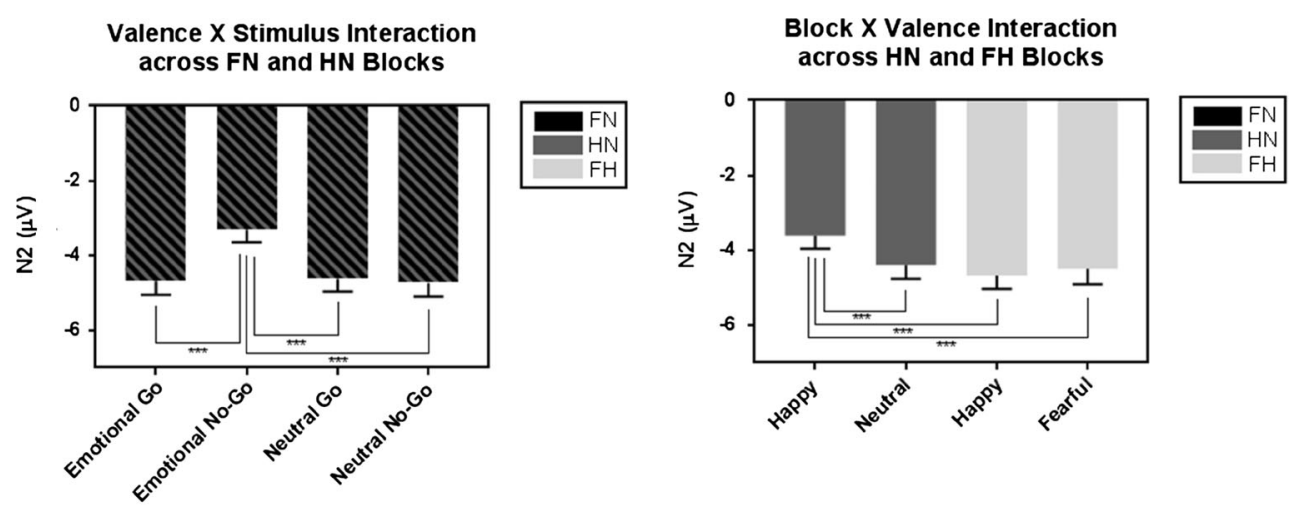

Block X Valence $X$ Stimulus Interaction across $\mathrm{FN}$ and $\mathrm{FH}$ Blocks

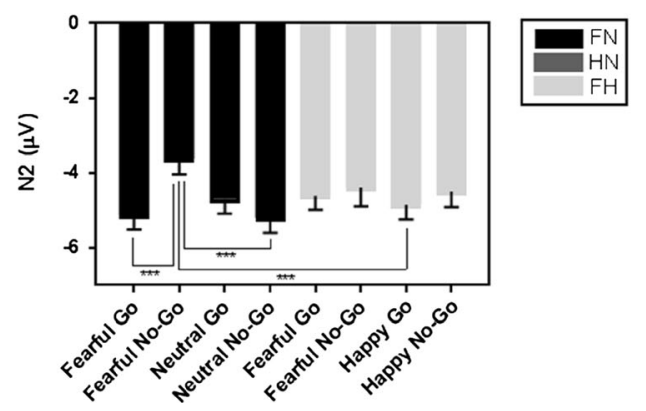

Fig. 5 The N2 was sensitive to emotional context: amplitudes were smaller to emotional no-go faces compared to neutral no-go or any type of go faces (top left). More specifically, cognitive control was disrupted

and discrimination for no-go versus go faces and for emotional versus neutral faces. In particular, fearful faces elicited greater N170 amplitudes compared to neutral and happy faces.

N2 Fear-neutral versus happy-neutral. The significant Valence $\times$ Stimulus interaction showed that N2 amplitudes were smaller for emotional no-go faces compared to neutral no-go or any type of go face, $F(1,65)=20.77, p<.001, \eta_{\mathrm{p}}{ }^{2}$ $=.24 ; p \mathrm{~s}<.001$ (see Fig. 5). There was also a main effect of block such that N2 amplitudes were larger in the fear-neutral versus happy-neutral block, $F(1,65)=12.81, p=.001, \eta_{\mathrm{p}}{ }^{2}$ $=.17$.

Fear-neutral versus fear-happy. The significant Block $\times$ Valence $\times$ Stimulus interaction showed that across and within blocks, N2 amplitudes were smaller to fearful no-go faces compared to fearful go, neutral no-go, and happy go faces, $F(1,65)$ $=9.41, p=.003, \eta_{\mathrm{p}}{ }^{2}=.13 ; p s<.01$.

Happy-neutral versus fear-happy. The significant main effect of stimulus showed that $\mathrm{N} 2$ amplitudes were smaller to no-go versus go faces, $F(1,65)=8.86, p=.004, \eta_{\mathrm{p}}{ }^{2}=.12$. The significant Block $\times$ Valence interaction further showed that N2 amplitudes were smallest to happy faces in the happy-neutral block compared to any other type of face across and within blocks ( $p s<.001), F(1$, $65)=11.54, p=.001, \eta_{\mathrm{p}}{ }^{2}=.15$.

N2 Summary. N2 amplitudes were sensitive to stimulus, valence, and block, indicating dampened N2 responses to no- by happy faces in the happy-neutral block (top right), and fearful no-go faces in the fear-neutral block (bottom). Note. FN = fear-neutral block; $\mathrm{HN}$ = happy-neutral block; FH = fear-happy block

go versus go faces, and emotional versus neutral faces. Specifically, fearful no-go faces elicited smaller N2 amplitudes compared to any other type of face.

\section{Associations between behavioral performance and ERP responses to face cues}

To test whether behavioral performance was related to ERP metrics of emotional context sensitivity, we conducted correlations between residual scores for behavioral measures ( $d$-prime, hit rate, false-alarm rate) to happy faces (vs. neutral faces) or fearful faces (vs. neutral or happy faces) and residual scores for ERPs (N170 and N2). $D$-prime scores were compared to ERPs for both go and no-go face cues. Hit rates were compared only to ERPs for go cues, while false-alarm rates were compared to only no-go cues.

Significant correlations emerged in the fear-neutral block only. ${ }^{7}$ Greater $d$-prime scores $(r=-.292, p=.017)$ and hit rates $(r=-.264, p=.032)$ for fearful versus neutral faces was related to greater amplitude N170 to fearful versus neutral go faces.

\footnotetext{
${ }^{7}$ For correlations, multiple comparisons were corrected using the BenjaminiHochberg procedure (Benjamini \& Hochberg, 1995), which involves ranking $p$ values and accounts for the number of tests conducted. This correction was applied separately to each family of regressions (i.e., separately for each behavioral measure), since this approach assumes sample independence. Raw $p$ values are reported and were significant using a false discovery rate criterion of 0.15 (Benjamini \& Hochberg, 1995).
} 
Table 2 Correlations among self-report questionnaires and residuals for hit rate, false-alarm rate, and $d$-prime

\begin{tabular}{|c|c|c|c|c|}
\hline & BDI & BAI & FREE & PACT \\
\hline \multicolumn{5}{|c|}{$D$-prime residuals } \\
\hline FN block & .071 & .034 & -.233 & -.045 \\
\hline HN block & $-.265^{*}$ & -.163 & .113 & .197 \\
\hline FH block & -.144 & -.072 & -.039 & .192 \\
\hline \multicolumn{5}{|c|}{ Hit rate residuals } \\
\hline FN block & -.028 & $-.232^{+}$ & .020 & .107 \\
\hline HN block & $-.312^{*}$ & $-.296^{*}$ & .088 & .145 \\
\hline FH block & -.127 & -.130 & -.144 & .061 \\
\hline \multicolumn{5}{|c|}{ False-alarm rate residuals } \\
\hline FN block & -.106 & .012 & -.140 & $-.307 *$ \\
\hline HN block & .076 & .035 & -.032 & -.070 \\
\hline FH block & .059 & -.020 & $-.255^{*}$ & .085 \\
\hline
\end{tabular}

Note. $\mathrm{N}=$ neutral; $\mathrm{F}=$ fear; $\mathrm{H}=$ happy. ${ }^{+} p<.10,{ }^{*} p<.05$

\section{Links between behavioral performance and emotional flexibility and well-being}

To test whether behavioral metrics of emotional context sensitivity relate to regulatory flexibility and efficacy, correlations were conducted between residual scores for behavioral measures ( $d$-prime, hit rate, and false-alarm rate) and self-report measures of emotional well-being and flexibility (see Table 2). Expressive flexibility was measured using the FREE (Burton \& Bonanno, 2015), coping flexibility was measured using the PACT (Bonanno, Pat-Horenczyk, et al., 2011), and depression and anxiety symptoms were measured using the BDI (Beck et al., 1996) and BAI (Beck et al., 1988), respectively.

Emotional well-being Beck's Depression Inventory (BDI) and Beck's Anxiety Inventory (BAI). Greater $d$-prime scores $(r=-.265, p=.032)$, and greater hit rates to happy versus neutral faces $(r=-.312, p=.011)$, were both related to lower depressive symptoms. Greater hit rates for happy $(r=-.296, p$ $=.016)$ and fearful $(r=-.232, p=.060)$ versus neutral faces were also associated with lower anxiety.

Emotion regulation and coping flexibility Flexible Regulation of Emotional Expression Scale (FREE). Expressive flexibility was associated with lower false-alarm rates for fear versus happy faces $(r=-.255, p=.039)$.

Perceived Ability to Cope with Trauma Scale (PACT) Coping flexibility was associated with lower false-alarm rates to fearful versus neutral faces $(r=-.307, p=.012)$.

\section{Links between ERPs and emotional flexibility and well-being}

To test whether ERP metrics of emotional context sensitivity relate to ER flexibility and efficacy, associations between residual scores for ERPs (N170 and N2) and self-report measures of emotional well-being and flexibility were examined (see Table 3).

Emotional well-being Beck's Depression Inventory ( $B D I)$ and Beck's Anxiety Inventory (BAI). Greater N170 to fearful versus happy no-go faces was associated with lower depressive symptoms $(r=.342, p=.005)$, whereas greater $\mathrm{N} 2$ to fearful versus happy go faces were associated with lower depression $(r=.270, p=.028)$ and anxiety symptoms $(r=.312$, $p=.011)$.

Emotion regulation and coping flexibility Flexible Regulation of Emotional Expression Scale (FREE). Expressive flexibility was associated with greater N170 to fearful versus neutral no-go faces $(r=-.375, p=.002)$, and greater $\mathrm{N} 2$ to happy versus neutral no-go faces $(r=-.253, p=$ $.040)$.

\section{Discussion}

The current findings demonstrate that measures of context sensitivity assessed during an emotional go/no-go task are

Table 3 Correlations among self-report questionnaires and residuals for N170 and N2

\begin{tabular}{clcccc}
\hline \multicolumn{1}{c}{} & & BDI & BAI & FREE & PACT \\
\hline $\begin{array}{c}\text { N170 residuals } \\
\text { FN block }\end{array}$ & Fear no-go & .176 & .020 & $-.375^{* *}$ & -.084 \\
& Fear go & .149 & -.041 & -.086 & -.089 \\
HN block & Happy no-go & .101 & -.012 & .041 & -.023 \\
& Happy go & -.067 & .196 & .174 & .117 \\
FH block & Fear no-go & $.342^{* *}$ & .037 & -.168 & .218 \\
& Fear go & -.103 & -.103 & -.089 & .126 \\
N2 residuals & & & & & \\
FN block & Fear no-go & .129 & -.046 & .125 & .015 \\
& Fear go & .066 & .177 & -.165 & .096 \\
HN block & Happy no-go & .047 & .077 & $-.253 *$ & -.039 \\
& Happy go & .123 & -.101 & -.033 & -.043 \\
FH block & Fear no-go & -.095 & -.059 & .133 & -.042 \\
& Fear go & $.270^{*}$ & $.312^{*}$ & -.054 & -.056 \\
\hline
\end{tabular}

Note. BDI = Beck's Depression Inventory; BAI = Beck's Anxiety Inventory; FREE = Flexible Regulation of Emotional Expression Scale; PACT $=$ Perceived Ability to Cope with Trauma Scale; $\mathrm{N}=$ neutral; $\mathrm{F}=$ fear; $\mathrm{H}=$ happy. $* p<.05, * * p<.01$ 
systematically correlated with emotional flexibility and wellbeing. Taken together, findings provide some of the first evidence linking behavioral and neurocognitive sensitivity to emotional context with broader dimensions of regulatory flexibility. Furthermore, the ability to harness and overcome the respective influence of pleasant and unpleasant emotional contexts may be important predictors of emotional wellbeing that should be distinguished from context-independent discrete strategy use. Importantly, although there were limited associations between behavioral performance and neurocognitive measures, the current findings indicate that behavioral and ERP metrics capture distinct aspects of context sensitivity. This highlights the importance of using neurocognitive methods to examine the time course of context-sensitive attentional processes, which may not be directly reflected in behavioral performance during the task but nevertheless relates to emotional flexibility and well-being.

Our findings illustrated that emotional context directly influenced behavioral performance. As predicted, happy faces facilitated approach to the go stimulus as reflected by greater $d$-prime scores than in both neutral and fearful contexts. Further, our secondary analyses of hit rates showed that this context sensitivity to happy faces was likely driven by greater hit rates more so that reduced false-alarm rates. Also, fearful faces hindered inhibition, as demonstrated by decreased $d$ prime scores when fearful faces were the no-go stimulus in both neutral and happy contexts. False-alarm rates were greater for emotional faces in general, indicating that context sensitivity in these cases may be driven by a disruption in inhibition by emotion more so than facilitation of hit rates by neutral faces. Thus, the emotional go/no-go task, as demonstrated in previous studies (Elliott, Rubinsztein, Sahakian, \& Dolan, 2000; Schulz et al., 2007; Shafritz, Collins, \& Blumberg, 2006; Tottenham et al., 2011), provides a multifaceted way to capture emotional context sensitivity.

Examination of ERPs provided an additional, and temporally sensitive, measure of emotional context sensitivity. Behavioral performance was correlated with ERP measures, but only in the fear-neutral block, perhaps since the difference between fearful and neutral faces was the most salient among the emotional context pairings in the task, resulting in more closely aligned behavioral and neurocognitive responding. Overall, however, emotional context elicited heightened early discrimination and selection (N170) but disrupted later cognitive control (N2) (consistent with Yang et al., 2014). Specifically, fearful faces bolstered early attentional selection and discrimination, as indicated by greater N170 amplitudes in neutral and happy contexts. Also, N170 amplitudes were greater for no-go versus go stimuli in contexts where both emotional and neutral faces were presented (fear-neutral and happy-neutral blocks). In contrast, inhibitory control, as indicated by $\mathrm{N} 2$ amplitudes, was blunted in response to fearful no-go versus go stimuli in both happy and neutral contexts, suggesting reduced recruitment of cognitive control resources. Both types of emotional no-go faces elicited reduced N2 amplitudes in neutral contexts. Interestingly, happy faces as both go and no-go stimuli blunted N2 responses in the happy-neutral block, suggesting that even pleasant emotions may interrupt cognitive control when presented in a context of neutral stimuli.

A key goal of the current study was to examine these biobehavioral indices of emotional context sensitivity in relation to self-reported adaptive functioning. In previous studies, individuals with symptoms of mood disorders have shown reduced behavioral facilitation in emotionally unpleasant contexts (Ladouceur et al., 2006). In the current study, as predicted, patterns of association emerged between behavioral performance during the emotional go/ no-go task and both emotional flexibility and well-being. Greater hit rates and $d$-prime scores to happy faces in a neutral context were associated with lower depression and anxiety. Consistent with Ladouceur et al. (2006), this suggests that, among those experiencing depression and anxiety symptoms, successful behavioral facilitation may be dampened for pleasant targets in a neutral context which can be viewed as emotionally unpleasant among individuals with mood disorders (e.g., Leppänen, Milders, Bell, Terriere, \& Hietanen, 2004).

Greater false-alarm rates to fearful and happy faces in neutral contexts were associated with lower coping flexibility and lower emotional expressive flexibility, respectively. Taken together, these findings suggest that the ability to overcome the potentially disruptive influence of emotional cues on inhibitory control is related to flexibility capacity. Also, ERPs reflecting early attentional selection and discrimination and later cognitive control during the emotional go/no-go task were related to emotional flexibility and well-being. In previous studies, symptoms of mood and attentional disorders have been associated with reduced N170s in emotional contexts (see Feuerriegel et al., 2015, for a review), and attenuated N2 amplitudes following no-go trials (Dong et al., 2010; Pliszka et al., 2000). In the current study, greater N170 amplitudes to fearful no-go faces was related to lower depression and greater reported emotional flexibility, suggesting that the ability to overcome the potentially disruptive impact of unpleasant stimuli on early attentional processes is associated with better mental health and emotion regulation. In addition, greater N2 amplitudes to happy versus neutral no-go faces were associated with greater emotional flexibility. Finally, enhanced N2 amplitudes to fearful go faces were associated with lower depression and anxiety, suggesting that those able to maintain higher magnitude N2 in fearful emotional contexts may be less vulnerable to symptoms of mood disorders.

While the current study established that biobehavioral responses were sensitive to emotional context and associated 
with self-reported emotional well-being and flexibility, some limitations should be noted. First, to avoid missing potentially meaningful relationships in hypothesis-driven analyses, corrections for multiple comparisons were not used in correlation analyses linking performance in the go/no-go task and selfreport measures. Since effect sizes were all approximately medium, these relationships should be interpreted as a first step in examining these associations, and serve as a basis for more targeted questions in future studies. Also, the emotional go/no-go task did not include angry or sad facial stimuli, which previous studies (e.g. Elliott et al., 2000; Schulz et al., 2007; Shafritz et al., 2006; Tottenham et al., 2011) show might influence task performance in distinct ways. For example, unpleasant emotions do not elicit avoidance responses in every context. Anger is considered an approach-related emotion, particularly in social contexts involving dominance motivations (Bossuyt, Moors, \& De Houwer, 2014; Carver \& Harmon-Jones, 2009). The design of the current study limits the generalization of the findings to only happy and fearful emotional contexts. Future research should elaborate on these findings by investigating whether sensitivity to a wider range or emotional contexts is related to even greater regulatory flexibility.

Also, while biobehavioral responses reflecting context sensitivity were associated with emotional well-being, including anxiety and depressive symptoms, the current study used a normative sample. Future research should aim to recruit clinically anxious or clinically depressed participants to examine disruptions of behavioral facilitation and inhibitory control among individuals with more severe psychopathological symptoms. Specifically, individuals with more severe anxiety symptoms may show either more extreme disruption of behavioral performance in an emotional context (due to attentional avoidance of emotion; e.g., Heuer, Rinck, \& Becker, 2007; Mansell, Clark, Ehlers, \& Chen, 1999), or no disruption at all (due to a hypervigilance to emotion; e.g. Bradley, Mogg, White, Groom, \& Bono, 1999; Williams, Mathews, \& MacLeod, 1996). A sample including more participants with severe symptoms could establish whether the relationship between behavioral performance disruption during the go/no-go task and self-reported anxiety and depression was linear (extreme disruption related to severe symptoms), or curvilinear (no disruption or extreme disruption related to severe symptoms). Also, since previous research has established that anxiety and depression are associated with insensitivity to emotional context (e.g., Coifman \& Bonanno, 2010; Gehricke \& Shapiro, 2000; Rottenberg et al., 2002), future research with this paradigm should also examine other aspects of regulatory flexibility beyond emotional context sensitivity, including the ability to use a diverse repertoire of ER strategies and to respond to feedback (Birk \& Bonanno, 2016).
Taken together, our results demonstrate that identification of the neurocognitive and behavioral patterns underlying regulatory flexibility will allow for a greater understanding of associations between emotion regulation and mental health, beyond a strict adaptive versus maladaptive dichotomy. Indeed, these biobehavioral measures of emotional context sensitivity may be signatures of mechanisms underlying the emergence and course of psychopathology. Future clinical research may benefit from using these metrics to assess or predict clinical course or outcomes as well as key aspects of emotion regulatory flexibility and functioning.

\section{References}

Aldao, A. (2013). The future of emotion regulation research capturing context. Perspectives on Psychological Science, 8(2), 155-172.

Ashley, V., Vuilleumier, P., \& Swick, D. (2004). Time course and specificity of event-related potentials to emotional expressions. NeuroReport, 15(1), 211-216.

Batty, M., \& Taylor, M. J. (2003). Early processing of the six basic facial emotional expressions. Cognitive Brain Research, 17(3), 613-620.

Beck, A. T., Epstein, N., Brown, G., \& Steer, R. A. (1988). An inventory for measuring clinical anxiety: Psychometric properties. Journal of Consulting and Clinical Psychology, 56(6), 893.

Beck, A. T., Steer, R. A., \& Brown, G. K. (1996). Beck Depression Inventory-II. San Antonio: Psychological Corporation.

Benjamini, Y., \& Hochberg, Y. (1995). Controlling the false discovery rate: a practical and powerful approach to multiple testing. Journal of the Royal Statistical Society. Series B (Methodological), 57, 289-300.

Bentin, S., Allison, T., Puce, A., Perez, E., \& McCarthy, G. (1996). Electrophysiological studies of face perception in humans. Journal of Cognitive Neuroscience, 8(6), 551-565.

Birk, J. L., \& Bonanno, G. A. (2016). When to throw the switch: The adaptiveness of modifying emotion regulation strategies based on affective and physiological feedback. Emotion, 16, 657-670.

Bokura, H., Yamaguchi, S., \& Kobayashi, S. (2001). Electrophysiological correlates for response inhibition in a go/nogo task. Clinical Neurophysiology, 112(12), 2224-2232.

Bonanno, G. A., \& Burton, C. L. (2013). Regulatory flexibility an individual differences perspective on coping and emotion regulation. Perspectives on Psychological Science, 8(6), 591-612.

Bonanno, G. A., Papa, A., Lalande, K., Westphal, M., \& Coifman, K. (2004). The importance of being flexible the ability to both enhance and suppress emotional expression predicts long-term adjustment. Psychological Science, 15(7), 482-487.

Bonanno, G. A., Pat-Horenczyk, R., \& Noll, J. (2011). Coping flexibility and trauma: The Perceived Ability to Cope With Trauma (PACT) scale. Psychological Trauma: Theory, Research, Practice, and Policy, 3(2), 117.

Bonanno, G. A., Westphal, M., \& Mancini, A. D. (2011). Resilience to loss and potential trauma. Annual Review of Clinical Psychology, 7, $511-535$.

Bossuyt, E., Moors, A., \& De Houwer, J. (2014). On angry approach and fearful avoidance: The goal-dependent nature of emotional approach and avoidance tendencies. Journal of Experimental Social Psychology, 50, 118-124.

Bradley, B. P., Mogg, K., White, J., Groom, C., \& Bono, J. (1999). Attentional bias for emotional faces in generalized anxiety disorder. British Journal of Clinical Psychology, 38(3), 267-278.

Burton, C. L., \& Bonanno, G. A. (2015). Measuring ability to enhance and suppress emotional expression: The Flexible Regulation of 
Emotional Expression (FREE) Scale. Psychological Assessment, 28(8), 929-941.

Bush, G., Luu, P., \& Posner, M. I. (2000). Cognitive and emotional influences in anterior cingulate cortex. Trends in Cognitive Sciences, 4(6), 215-222.

Carver, C. S., \& Harmon-Jones, E. (2009). Anger is an approach-related affect: Evidence and implications. Psychological Bulletin, 135(2), 183

Carver, C. S., \& White, T. L. (1994). Behavioral inhibition, behavioral activation, and affective responses to impending reward and punishment: The BIS/BAS scales. Journal of Personality and Social Psychology, 67(2), 319.

Casey, B., Trainor, R. J., Orendi, J. L., Schubert, A. B., Nystrom, L. E., Giedd, J. N.,... Cohen, J. D. (1997). A developmental functional MRI study of prefrontal activation during performance of a go-nogo task. Journal of Cognitive Neuroscience, 9(6), 835-847.

Chang, C. (2001). The impacts of emotion elicited by print political advertising on candidate evaluation. Media Psychology, 3(2), 91118.

Coifman, K. G., \& Bonanno, G. A. (2010). When distress does not become depression: Emotion context sensitivity and adjustment to bereavement. Journal of Abnormal Psychology, 119(3), 479.

Cole, P. M., Martin, S. E., \& Dennis, T. A. (2004). Emotion regulation as a scientific construct: Methodological challenges and directions for child development research. Child Development, 75(2), 317-333.

Davidson, R. J., Putnam, K. M., \& Larson, C. L. (2000). Dysfunction in the neural circuitry of emotion regulation-A possible prelude to violence. Science, 289(5479), 591-594.

Diminich, E. D., \& Bonanno, G. A. (2014). Faces, feelings, words: Divergence across channels of emotional responding in complicated grief. Journal of Abnormal Psychology, 123(2), 350.

Dong, G., Lu, Q., Zhou, H., \& Zhao, X. (2010). Impulse inhibition in people with Internet addiction disorder: Electrophysiological evidence from a go/no-go study. Neuroscience Letters, 485(2), 138142 .

Eimer, M. (2011). The face-sensitivity of the 170 component. Frontiers in Human Neuroscience, 5, 119.

Elliott, R., Rubinsztein, J. S., Sahakian, B. J., \& Dolan, R. J. (2000). Selective attention to emotional stimuli in a verbal go/nogo task: An fMRI study. NeuroReport, 11(8), 1739-1744.

Falkenstein, M., Hoormann, J., \& Hohnsbein, J. (1999). ERP components in go/no-go tasks and their relation to inhibition. Acta Psychologica, 101(2), 267-291.

Feuerriegel, D., Churches, O., Hofmann, J., \& Keage, H. A. (2015). The N170 and face perception in psychiatric and neurological disorders: A systematic review. Clinical Neurophysiology, 126(6), 1141-1158.

Geczy, I., Czigler, I., \& Balazs, L. (1998). Effects of cue information on response production and inhibition measured by event-related potentials. Acta Physiologica Hungarica, 86(1), 37-44.

Gehricke, J.-G., \& Shapiro, D. (2000). Reduced facial expression and social context in major depression: Discrepancies between facial muscle activity and self-reported emotion. Psychiatry Research, 95(2), 157-167.

Gray, J. R., \& Braver, T. S. (2002). Integration of emotion and cognitive control. Advances in Consciousness Research, 44, 289-316.

Gross, J. J., \& Levenson, R. W. (1993). Emotional suppression: Physiology, self-report, and expressive behavior. Journal of Personality and Social Psychology, 64(6), 970.

Gupta, S., \& Bonanno, G. A. (2011). Complicated grief and deficits in emotional expressive flexibility. Journal of Abnormal Psychology, 120(3), 635.

Heuer, K., Rinck, M., \& Becker, E. S. (2007). Avoidance of emotional facial expressions in social anxiety: The approach-avoidance task. Behaviour Research and Therapy, 45(12), 2990-3001.
Itier, R. J., \& Taylor, M. J. (2004). N170 or N1? Spatiotemporal differences between object and face processing using ERPs. Cerebral Cortex, 14(2), 132-142.

John, O. P., \& Gross, J. J. (2004). Healthy and unhealthy emotion regulation: Personality processes, individual differences, and life span development. Journal of Personality, 72(6), 1301-1334.

Kashdan, T. B., \& Rottenberg, J. (2010). Psychological flexibility as a fundamental aspect of health. Clinical Psychology Review, 30(7), 865-878.

Kiefer, M., Marzinzik, F., Weisbrod, M., Scherg, M., \& Spitzer, M. (1998). The time course of brain activations during response inhibition: Evidence from event-related potentials in a go/no go task. NeuroReport, 9(4), 765-770.

Kopp, B., Mattler, U., Goertz, R., \& Rist, F. (1996). N2, P3 and the lateralized readiness potential in a nogo task involving selective response priming. Electroencephalography and Clinical Neurophysiology, 99(1), 19-27.

Krombholz, A., Schaefer, F., \& Boucsein, W. (2007). Modification of N170 by different emotional expression of schematic faces. Biological Psychology, 76(3), 156-162.

Ladouceur, C. D., Dahl, R. E., Williamson, D. E., Birmaher, B., Axelson, D. A., Ryan, N. D., \& Casey, B. (2006). Processing emotional facial expressions influences performance on a go/no-go task in pediatric anxiety and depression. Journal of Child Psychology and Psychiatry, 47(11), 1107-1115.

Leppänen, J. M., Milders, M., Bell, J. S., Terriere, E., \& Hietanen, J. K. (2004). Depression biases the recognition of emotionally neutral faces. Psychiatry Research, 128(2), 123-133.

Mansell, W., Clark, D. M., Ehlers, A., \& Chen, Y.-P. (1999). Social anxiety and attention away from emotional faces. Cognition \& Emotion, 13(6), 673-690.

Park, M., Chang, E. R., \& You, S. (2015). Protective role of coping flexibility in PTSD and depressive symptoms following trauma. Personality and Individual Differences, 82, 102-106.

Pizzagalli, D. A., Lehmann, D., Hendrick, A. M., Regard, M., PascualMarqui, R. D., \& Davidson, R. J. (2002). Affective judgments of faces modulate early activity $(\sim 160 \mathrm{~ms})$ within the fusiform gyri. NeuroImage, 16(3), 663-677.

Pliszka, S. R., Liotti, M., \& Woldorff, M. G. (2000). Inhibitory control in children with attention-deficit/hyperactivity disorder: Event-related potentials identify the processing component and timing of an impaired right-frontal response-inhibition mechanism. Biological Psychiatry, 48(3), 238-246.

Rottenberg, J., Gross, J. J., \& Gotlib, I. H. (2005). Emotion context insensitivity in major depressive disorder. Journal of Abnormal Psychology, 114(4), 627.

Rottenberg, J., Kasch, K. L., Gross, J. J., \& Gotlib, I. H. (2002). Sadness and amusement reactivity differentially predict concurrent and prospective functioning in major depressive disorder. Emotion, 2(2), 135.

Schulz, K. P., Fan, J., Magidina, O., Marks, D. J., Hahn, B., \& Halperin, J. M. (2007). Does the emotional go/no-go task really measure behavioral inhibition?: Convergence with measures on a non-emotional analog. Archives of Clinical Neuropsychology, $22(2), 151-160$

Shafritz, K. M., Collins, S. H., \& Blumberg, H. P. (2006). The interaction of emotional and cognitive neural systems in emotionally guided response inhibition. NeuroImage, 31(1), 468-475.

Sheppes, G., Catran, E., \& Meiran, N. (2009). Reappraisal (but not distraction) is going to make you sweat: Physiological evidence for self-control effort. International Journal of Psychophysiology, 71(2), 91-96.

Tamir, M. (2009). What do people want to feel and why? Pleasure and utility in emotion regulation. Current Directions in Psychological Science, 18(2), 101-105. 
Tottenham, N., Hare, T. A., \& Casey, B. (2011). Behavioral assessment of emotion discrimination, emotion regulation, and cognitive control in childhood, adolescence, and adulthood. Frontiers in Psychology, 2(39), 2.

Tottenham, N., Tanaka, J. W., Leon, A. C., McCarry, T., Nurse, M., Hare, T. A., ... Nelson, C. (2009). The NimStim set of facial expressions: Judgments from untrained research participants. Psychiatry Research, 168(3), 242-249.

Urry, H. L. (2009). Using reappraisal to regulate unpleasant emotional episodes: Goals and timing matter. Emotion, 9(6), 782.

Van Veen, V., \& Carter, C. S. (2002). The timing of action-monitoring processes in the anterior cingulate cortex. Journal of Cognitive Neuroscience, 14(4), 593-602.

Wang, X.-W., Nie, D., \& Lu, B.-L. (2014). Emotional state classification from EEG data using machine learning approach. Neurocomputing, 129, 94-106.
Webb, T. L., Miles, E., \& Sheeran, P. (2012). Dealing with feeling: A meta-analysis of the effectiveness of strategies derived from the process model of emotion regulation. Psychological Bulletin, $138(4), 775$

Weinberg, A., Venables, N. C., Proudfit, G. H., \& Patrick, C. J. (2015). Heritability of the neural response to emotional pictures: Evidence from ERPs in an adult twin sample. Social Cognitive and Affective Neuroscience, 10(3), 424-434.

Westphal, M., Seivert, N. H., \& Bonanno, G. A. (2010). Expressive flexibility. Emotion, 10(1), 92.

Williams, J. M. G., Mathews, A., \& MacLeod, C. (1996). The emotional Stroop task and psychopathology. Psychological Bulletin, 120(1), 3.

Yang, S., Luo, W., Zhu, X., Broster, L. S., Chen, T., Li, J., \& Luo, Y. (2014). Emotional content modulates response inhibition and perceptual processing. Psychophysiology, 51(11), 1139-1146. 